\title{
RAMIFICATIONS, OLD AND NEW, OF THE EIGENVALUE PROBLEM
}

\section{HERMANN WEYL}

Since this is a lecture dedicated to the memory of Josiah Willard Gibbs let me start with that purely mathematical discovery which Gibbs contributed to the theory of Fourier series. Fourier series have to do with the eigenvalues and eigenfunctions of the oldest, simplest, and most important of all spectrum problems, that of the vibrating string. In preparing this lecture, the speaker has assumed that he is expected to talk on a subject in which he had some first-hand experience through his own work. And glancing back over the years he found that the one topic to which he has returned again and again is the problem of eigenvalues and eigenfunctions in its various ramifications. It so happens that right at the beginning of my mathematical career I wrote two papers on what we now call the Gibbs phenomenon.

1. Gibbs phenomenon. Take a simple periodic function with a discontinuity, for example, the function $1^{\circ}(x)$ of period $2 \pi$ which equals 0 for $-\pi<x<0$ and 1 for $0<x<\pi$. In a letter to the editor of Nature published on April 27, 1899, Gibbs, correcting a statement in a previous letter, pointed out that the limit of the graphs of the partial sums $y=1_{n}^{0}(x)$ of the Fourier series of $1^{0}(x)$ includes not only the vertical ascent from the level 0 to the level 1 at $x=0$, but extends vertically beyond it by a specific amount. A. A. Michelson had started the discussion in Nature by criticizing the way in which the mathematicians are wont to describe the limit of the sequence of those partial sums; he had pleaded for adding to the two horizontal levels the vertical precipice. Today we find in the notion of uniform convergence the most adequate analysis of the phenomenon. Introduce the sinus integral

$$
\operatorname{Si}(x)=\frac{1}{\pi} \int_{-\infty}^{x} \frac{\sin \xi}{\xi} d \xi
$$

and consider a closed interval $I$, say $-\pi / 2 \leqq x \leqq \pi / 2$, containing only the one discontinuity at $x=0$. It is, of course, not true that the difference between $1^{\circ}(x)$ and the $n$th partial sum $1_{n}^{0}(x)$ converges

The twenty-second Josiah Willard Gibbs lecture delivered at Columbus, Ohio, December 28, 1948, under the auspices of the American Mathematical Society; received by the editors January 17, 1949. 
uniformly to zero in $I$, but it is true that $1_{n}^{0}(x)-\mathrm{Si}(n x)$ does so. Thus the graph of $1_{n}^{0}(x)$ for large $n$ is essentially that of the undulating function $\mathrm{Si}(x)$ compressed at the ratio $1: n$ in the $x$-direction.

Instead of direct summation one can apply to infinite series, in particular to Fourier series, other methods of summation. Let me mention only one here: in the Fourier series $\sum_{n=-\infty}^{\infty} a_{n} \mathrm{e}^{i n x}$ add the factor $\mathrm{e}^{-n^{2} t}$ to the $n$th term and then let the positive parameter $t=$ time in the resulting sum

$$
\sum_{n} a_{n} \mathrm{e}^{-n^{2} t} \mathrm{e}^{i n x}
$$

converge to zero. Since that sum is a solution of the equation of heat conduction

$$
\partial^{2} u / \partial x^{2}-\partial u / \partial t=0,
$$

I call this the heat conduction summation. The Gibbs phenomenon for this summation is ruled by the function

$$
\operatorname{Er}(x)=\frac{1}{\pi^{1 / 2}} \int_{-\infty}^{x} \mathrm{e}^{-\xi^{2}} d \xi
$$

in the same sense as it is ruled by $\mathrm{Si}(x)$ for the direct summation. This is an immediate consequence of the fact that $\mathrm{Er}\left(x / 2 t^{1 / 2}\right)$ is actually a solution-though not a periodic one-of the heat equation.

In the two papers just mentioned [ 1$]^{1}$ I considered the Gibbs phenomenon for a certain general type of summation methods. My chief concern was with the simplest two-dimensional case of eigenfunctions, namely Laplace's expansion of functions on a sphere in terms of spherical harmonics. Nothing new occurs if the function has a discontinuity along a smooth line with a continuous tangent. But quite an impressive mountain landscape develops in the neighborhood of a point where this line makes an angle. There was one specific one-dimensional problem which attracted my attention. It deals with a circular metal ring consisting of two halves of different conductivities $\alpha$ and $\beta$. Assume the normalization $\alpha+\beta=1$. If one of the halves has a temperature of $100^{\circ} \mathrm{C}$. at the time $t=0$, the other of $0^{\circ}$, how will the temperature level off in the progress of time? The solution can be easily expressed by means of the function $\mathrm{Er}$, and this gives the Gibbs phenomenon for the heat conduction summation of the corresponding eigenfunction expansion. The Gibbs phenomenon for direct sum-

1 The letters (A), (B), $\cdots$ refer to a number of notes printed at the end of the paper (and not included in the actual lecture as delivered at the Columbus meeting). The bold face numerals $[1], \cdots$ refer to the bibliography. 
mation is a more subtle question because the distribution of the eigenvalues $\lambda$ depends on the arithmetical character of the numbers $\alpha$ and $\beta$. Only if they are rational, the problem may be settled by fairly direct computation (A). The result would carry over to an arbitrary irrational $\alpha$ if it were true that $\alpha$ can be approximated by a sequence of fractions $a_{n} / c_{n}(n=1,2, \cdots)$ such that

$$
n\left(\alpha-a_{n} / c_{n}\right) \rightarrow 0, \quad c_{n} / n \rightarrow 0 \quad \text { for } n \rightarrow \infty .
$$

It is simple enough to show that this can be done, and thus to determine the Gibbs phenomenon for arbitrary conductivities $\alpha, \beta$. Our lemma on Diophantine approximations proves at the same time the equidistribution mod. 1 of the multiples $n \alpha$ of an irrational number $\alpha$.

When, not so long after, I learned through Felix Bernstein about the problem of mean motion in Lagrange's linear theory of perturbation for the planetary system, a problem P. Bohl had connected with that of equidistribution mod. 1 , I remembered this investigation and tried to settle the question of equidistribution in a more general form [2]. This is an example of how experience in one field of mathematics may give one the lead in an entirely different field. It is chiefly for this lesson that I have mentioned here this early work of mine, by which, in a very modest way, I paid homage to the genius of Gibbs about forty years ago.-Incidentally, Fourier series provided the basis for the analytic method which I brought to bear on the general problem of deciding whether a given sequence of real numbers $\xi_{1}, \xi_{2}, \ldots$ is equidistributed mod. 1 . Such equidistribution can be formulated as a statement concerning the mean value of any Riemann integrable function $f(x)$ of period 1 for the argument values $x=\xi_{1}, \xi_{2}, \ldots$; and the gist of the method lies in the observation that verification of the statement for the special periodic functions $\mathrm{e}^{2 \pi n i x}$ $(n=0, \pm 1, \pm 2, \cdots)$ is sufficient.

2. Limit circle and limit point. As one knows, Fourier series were generalized by Sturm and Liouville so as to cover the eigenvalues $\lambda$ and eigenfunctions $\phi(s)$ of the self-adjoint differential equation

$$
L_{\lambda}(\phi) \equiv\left\{\frac{d}{d s}\left(p(s) \frac{d \phi}{d s}\right)-q(s) \phi(s)\right\}+\lambda \phi(s)=0,
$$

subject to a real linear boundary condition at either end of the interval $0 \leqq s \leqq l$. The coefficients $p(s)>0$ and $q(s)$ are given real continuous functions in this interval. Let the abbreviation $\phi^{\prime}$ be used for $p(s) d \phi / d s$. A real linear boundary condition for $s=l$ is of the form 


$$
\phi^{\prime}(l)-h \cdot \phi(l)=0
$$

with a real constant $h$ (not excluding $h=\infty$ ). For a solution $\phi$ of $L_{\lambda}(\phi)=0$ and a solution $\phi_{*}$ of $L_{\lambda_{*}}\left(\phi_{*}\right)=0$ we have the simple Green's formula

$$
\left[\phi \phi_{*}^{\prime}-\phi_{*} \phi^{\prime}\right]_{0}^{l}=\left(\lambda-\lambda_{*}\right) \int_{0}^{l} \phi \phi_{*} d s .
$$

It shows that eigenfunctions $\phi, \phi_{*}$ belonging to two distinct eigenvalues $\lambda, \lambda_{*}$ are necessarily orthogonal, $\int_{0}^{l} \phi \phi_{*} \cdot d s=0$. On taking $\lambda_{*}=\bar{\lambda}, \phi_{*}=\bar{\phi}$ one finds that for a non-real $\lambda$ the function $\phi(s)$ cannot satisfy a real linear boundary condition at both ends without vanishing identically; for under these circumstances our equation would give $\int_{0}^{l} \phi \bar{\phi} d s=0$. The positive-definite character of the integrand $\phi \bar{\phi}$ is decisive here.

The spectrum of the eigenvalues is discrete provided the differential equation is regular at both ends, that is, provided $p(s)$, $q(s)$ are continuous and $p(s)$ actually positive throughout the closed interval $0 \leqq s \leqq l$. If we make this assumption only for the right-open interval $0 \leqq s<l$, as we shall now do, then the end $s=0$ stays regular, but the end $s=l$ is (possibly) singular. Let us throw the singular end into $s=+\infty$. Under these circumstances one must expect that a continuous spectrum will appear side by side with the point spectrum. Moreover it seems that sometimes a boundary condition is required at the singular end, just as it would be for a regular one, but sometimes not.

The very first result by which I added my mite to our stock of mathematical knowledge had to do with the clarification of this issue [3]. Since one cannot vouch that the spectrum will not cover the entire real $\lambda$-axis, I had the simple idea (not as trivial at that time as it has now become) to determine Green's function $G(s, t)$ neither for $\lambda=0$ nor for any real $\lambda$, but for a $\lambda$ in the upper half-plane, $5 \lambda>0$, for example for $\lambda=i$. Let $\eta(s), \theta(s)$ designate the two solutions $\phi(s)=\phi(s ; \lambda)$ of $(1)$ determined by the initial conditions

$$
\eta(0)=1, \eta^{\prime}(0)=0 \text { and } \theta(0)=0, \theta^{\prime}(0)=1
$$

respectively, and then consider the solution

$$
\phi(s)=w \cdot \eta(s)-\theta(s)
$$

which combines them by means of an arbitrary constant $w$. The question naturally arises for which values of $w$ this $\phi(s)$ satisfies a real linear boundary condition (2) at $s=i$. The answer is: for those $w$ 
that lie on a certain circle $C_{l}$ in the complex $w$-plane. Indeed the condition (2) gives

$$
\left(w \eta^{\prime}-\theta^{\prime}\right)-h(w \eta-\theta)=0 \quad \text { for } s=l
$$

or

$$
w=\frac{\theta^{\prime}(l)-h \theta(l)}{\eta^{\prime}(l)-h \eta(l)}
$$

and this fractional linear or Möbius transformation $h \rightarrow w$ maps the real $h$-axis upon a circle in the $w$-plane. Here $\lambda$ is a given value in the upper half-plane. We now compare two such values $\lambda, \lambda_{0}$. Whereas $\phi(s)=\phi(s ; \lambda), \eta, \theta$, and so on refer to $\lambda$, let $\phi_{0}, \eta_{0}, \theta_{0}$, and so on refer to $\lambda_{0}$. By picking a point $w^{0}$ on $C_{l}^{0}=C_{l}\left(\lambda_{0}\right)$ one fixes the coefficient $h$ of the real boundary condition (2). Clearly the point $w=w(\lambda)$ on $C_{l}=C_{l}(\lambda)$ for which (4) satisfies the same boundary condition as $\phi_{0}=w^{0} \cdot \eta_{0}-\theta_{0}$ at $s=l$ proceeds from $w^{0}$ by a certain Möbius transformation $w^{0} \rightarrow w$. Points $w^{0}$ and $w$ on $C_{l}^{0}$ and $C_{l}$ thus related may be called homologous points.

We now face the task of transferring these obvious answers to the limit $l \rightarrow \infty$. For that purpose the definitions of the circle $C_{l}$ and of the homology between the two circles $C_{l}\left(\lambda_{0}\right)$ and $C_{l}(\lambda)$ must first be given a new form, one that looks more complicated but is in fact more instructive. Put $\lambda_{*}=\bar{\lambda}, \phi_{*}=\bar{\phi}$ in (3):

$$
\left[\phi \Phi^{\prime}-\Phi \phi^{\prime}\right]_{0}^{l}=(\lambda-\bar{\lambda}) \cdot \int_{0}^{l} \phi \bar{\phi} d s .
$$

On account of this identity the requirement that $\phi$ satisfies a real linear boundary condition at $s=l, \phi \bar{\phi}^{\prime}-\bar{\phi} \phi^{\prime}=0$ for $s=l$, is equivalent to the relation

$$
\Im \lambda \int_{0}^{\imath} \phi \bar{\Phi} d s=\Im w \quad \text { for } \phi=w \eta-\theta .
$$

This is indeed the equation of a circle $C_{l}$ in the upper half $w$-plane. The points of the circular disk $\left(C_{l}\right)$ bounded by $C_{l}$ are characterized by the inequality

$$
\int_{0}^{l} \phi \bar{\phi} d s \leqq \Im w / J \lambda .
$$

This shows at once that $C_{l^{\prime}}$ lies inside $C_{l}$ if $l^{\prime}>l$. Hence with $l$ tending to infinity, $C_{l}$ shrinks either to a limit circle or a limit point $C=C(\lambda)$. This alternative, limit circle or limit point, is clearly the correct 
formulation of the question whether or not to impose a boundary condition at $s=\infty$.

Next we have to put the description of the homology mapping $w^{0} \rightarrow w$ of the circle $C_{l}^{0}$ upon $C_{l}$ into a form suitable for passage to the limit $l \rightarrow \infty$. Pick a point $w^{0}$ on $C_{l}^{0}=C_{l}\left(\lambda_{0}\right)$ and form

$$
\phi_{0}(s)=\phi\left(s ; \lambda_{0}\right)=w^{0} \cdot \eta\left(s ; \lambda_{0}\right)-\theta\left(s ; \lambda_{0}\right) .
$$

The solution

$$
\phi(s)=\phi(s ; \lambda)=w \cdot \eta(s ; \lambda)-\theta(s ; \lambda),
$$

satisfying at the end $s=l$ the same real linear boundary condition as $\phi_{0}(s)$, is obtained from $\phi_{0}(s)$ by solving the linear integral equation

$$
\phi_{0}(s)=\phi(s)-\left(\lambda-\lambda_{0}\right) \int_{0}^{l} G^{0}(s, t) \cdot \phi(t) d t
$$

the kernel $G^{0}(s, t)$ of which is Green's function for $\lambda_{0}$ :

$$
G^{0}(s, t)= \begin{cases}\phi_{0}(s) \cdot \eta_{0}(t) & (t \leqq s), \\ \eta_{0}(s) \cdot \phi_{0}(t) & (s \leqq t) .\end{cases}
$$

From the solution $\phi(s),(4)$, one gets $w=w(\lambda)$ as its initial value $\phi(0)$. This prescription at once carries over to the limit $l \rightarrow \infty$; one has simply to replace integration from 0 to $l$ in our integral equation by one extending from 0 to $\infty$. Of course in (5) the factor $w^{0}$ is now supposed to be a point on the circle $C^{0}$ (whether that is a real circle or degenerates into a point). Indeed, on trying to solve in the simplest way the integral equation thus resulting, namely by the Neumann series, one finds by direct estimates that the series converges within the circle around $\lambda_{0}$ in the $\lambda$-plane that touches the real axis. Hence analytic continuation encounters no obstacle, and $\phi(0 ; \lambda)=w(\lambda)$ is a regular analytic function in the entire upper half $\lambda$-plane. The method works in both the limit-circle and the limit-point cases. In the latter $w^{0}$ is the limit point for $\lambda_{0}$, and the construction gives the limit point $w=w(\lambda)$ for $\lambda$. Whether the singular end $s=\infty$ is of the limit circle or limit point type does not depend on the value of $\lambda$ as long as $\lambda$ is restricted to the upper half-plane. In the limit circle case it follows easily that the homology mapping $w^{0} \rightarrow w=w(\lambda)$ of $C^{0}$ onto $C$ is a Möbius transformation with coefficients depending analytically on $\lambda$ (for $5 \lambda>0$ ).

If one replaces the differential equation (1) by the corresponding difference equation, one arrives at a neat formulation for the theory of Stieltjes' continued fractions and his moment problem. It was treated 
by E. Hellinger in a manner analogous to the one outlined here for the differential equation [4]. The moment problem is a very special limiting case of an interpolation problem in the theory of analytic functions $w=w(\lambda)$ of a complex variable $\lambda$ first studied by G. Pick and R. Nevanlinna [5]. It concerns analytic functions $w(\lambda)$ defined in the upper half $\lambda$-plane, $J \lambda>0$, the values of which have themselves positive imaginary parts. For the moment let us call them positive functions. How far is such a function determined if its values $w\left(\alpha_{n}\right)$ are prescribed for a sequence of points $\lambda=\alpha_{1}, \alpha_{2}, \cdots$ in the upper half-plane? The differential problem corresponding to this interpolation or difference problem can be put in the form of a system of two linear differential equations of the first order for two unknowns $\phi, \phi^{\prime}$ containing the spectral parameter $\lambda$ in broken linear fashion,

$$
\left\{\begin{array}{l}
\frac{d \phi}{d s}=\frac{\lambda a_{1}(s)-b_{1}(s)}{\lambda a(s)-b(s)} \cdot \phi^{\prime}(s), \\
\frac{d \phi^{\prime}}{d s}=\frac{\lambda a_{2}(s)-b_{2}(s)}{\lambda a(s)-b(s)} \cdot \phi(s)
\end{array}\right.
$$

with real coefficients which satisfy the inequalities

$$
k^{\prime}(s)=a_{1}(s) b(s)-b_{1}(s) a(s)>0, k(s)=a(s) b_{2}(s)-b(s) a_{2}(s)>0 .
$$

This general system now replaces our former system (1) or

$$
\frac{d \phi}{d s}=\frac{1}{p(s)} \cdot \phi^{\prime}(s), \quad \frac{d \phi^{\prime}}{d s}=(q(s)-\lambda) \cdot \phi(s) .
$$

The theory of limit circles and of the homology mapping of the limit circles for different values of $\lambda$ carries over practically without alteration to this more general problem [6]. The decisive point is the positive definite character of the integrand

$$
\frac{k \phi \bar{\phi}+k^{\prime} \phi^{\prime} \phi^{\prime}}{(\lambda a-b)(\bar{\lambda} a-b)}
$$

that appears in Green's formula and takes over the role played by $\phi \bar{\phi}$ in the problem $\left(\mathrm{L}_{\lambda}\right)$. By direct constructive solutions of integral equations one thus proves the fundamental facts about the Nevanlinna interpolation problem, which Nevanlinna himself had derived with the aid of some of the strong "existential" methods characteristic for the theory of analytic functions, such as the Vitali theorem (B).

\section{Expansion theorem for ordinary self-adjoint linear differential}


equations of second order with singular end. For the classical problem $\left(\mathrm{L}_{\lambda}\right)$ the investigation of the singular end $s=\infty$ is merely a preliminary to the study of expansions by eigenfunctions. What one has to expect can be predicted when one first replaces the singular end $\infty$ by the regular end $l$. But one has to obliterate the feature of a discrete spectrum by writing the sum over the eigenvalues in the expansion formula as a Stieltjes integral involving a non-decreasing step function. Rather than attempt to carry out the passage to the limit $l \rightarrow \infty$ one seeks to verify the formula thus obtained directly for the interval $0 \leqq s<\infty$ with the singular end $\infty$. For simplicity's sake let us prescribe the boundary condition $\phi^{\prime}(0)=0$ at the regular end $s=0$. Then we know a priori that for any eigenvalue $\lambda=\lambda_{n}$ the function $\eta\left(s ; \lambda_{n}\right)$ must be the eigenfunction. The eigenvalues are those real values of $\lambda$ for which $\eta(s ; \lambda)$ satisfies a given real linear boundary condition $\eta^{\prime}(l ; \lambda)-h \cdot \eta(l ; \lambda)=0$ at the end $s=l$.

Choose a definite $\lambda_{0}$ with positive imaginary part, for example $\lambda_{0}=i$. Fixing the coefficient $h$ amounts to fixing a definite point $w^{0}$ on the circle $C_{l}\left(\lambda_{0}\right)=C^{0}$. The expansion of the arbitrary function $f(s)$ is then given by

$$
f(s) \sim \sum_{n} a_{n} \cdot \eta\left(s ; \lambda_{n}\right) \quad \text { where } \quad a_{n}=\int_{0}^{l} \eta\left(s ; \lambda_{n}\right) r_{l}\left(\lambda_{n}\right) f(s) d s
$$

and

$$
r_{l}\left(\lambda_{n}\right)=1 / \int_{0}^{l}\left(\eta\left(s ; \lambda_{n}\right)\right)^{2} d s
$$

Let $\Delta=\left(\lambda_{1}, \lambda_{2}\right)$ be any interval on the real $\lambda$-axis, and, $x(\lambda)$ being any function of the real $\lambda$, let $\Delta x$ stand for the difference $x\left(\lambda_{2}\right)-x\left(\lambda_{1}\right)$. We may now define a nondecreasing step function $\rho_{l}(\lambda)$ by the equation

$$
\Delta \rho_{l}=\sum_{\lambda_{n} \in \Delta} r_{l}\left(\lambda_{n}\right)
$$

(If one of the ends $\lambda_{1}, \lambda_{2}$ of the interval is an eigenvalue, the summand $r_{l}\left(\lambda_{1}\right)$ or $r_{l}\left(\lambda_{2}\right)$ in (7) should be counted with the weight $1 / 2$ only.) After forming

$$
\Delta \mathrm{P}(s)=\int_{\Delta} \eta(s ; \lambda) d \rho(\lambda), \quad \Delta \alpha=\int_{0}^{l} \Delta \mathrm{P}(s) \cdot f(s) d s
$$

our expansion appears as the following Stieltjes integral extending over the real $\lambda$-axis: 


$$
f(s) \sim \int_{-\infty}^{+\infty} \eta(s ; \lambda) d \alpha(\lambda) .
$$

One has good reason to hope that in this form the expansion theorem will carry over to the interval $(0, \infty)$ with the singular end $\infty$, and in anticipation of this result we have dropped the subscript $l$ in (8). The whole problem boils down to determining the nondecreasing function $\rho(\lambda)$.

For a finite interval $0 \leqq s \leqq l$ and a non-real $\lambda$ one easily proves (C) the following expansion, which is uniformly convergent with respect to $s$ :

$$
J \phi(s ; \lambda)=\int_{-\infty}^{+\infty} J \frac{1}{\mu-\lambda} \cdot \eta(s ; \mu) d \rho(\mu)
$$

in particular $(s=0)$

$$
J w(\lambda)=\int_{-\infty}^{+\infty} J \frac{1}{\mu-\lambda} \cdot d \rho(\mu) .
$$

We expect these equations to hold even for the infinite intervalalthough $\rho(\lambda)$ may then cease to be a step function-if $w(\lambda)$ and $\phi(s ; \lambda)$ are constructed from a (or the) point $w^{0}$ on the limit circle $C^{0}$ according to the prescription given before. Denote by $\Delta_{\epsilon}$ the segment $\Delta$ after it has been raised by the positive amount $\epsilon$ in the direction of the imaginary axis. One computes $\Delta \rho$ and $\Delta \mathrm{P}(s)=\int_{\Delta} \eta(s ; \lambda) d \rho(\lambda)$ from (11) and (10) as the limits

$$
\begin{aligned}
\Delta \rho & =\lim _{\epsilon \rightarrow 0} \frac{1}{\pi} \int_{\Delta_{\epsilon}} J w(\lambda) \cdot d \lambda, \\
\Delta \mathrm{P}(s) & =\lim _{\epsilon \rightarrow 0} \frac{1}{\pi} \int_{\Delta_{\epsilon}} J \phi(s ; \lambda) \cdot d \lambda .
\end{aligned}
$$

The first formula determines $\rho$.

When turning these heuristic arguments into an actual proof one should first endeavor to prove the existence of the limit (12) and then to establish the expansion (9), (8), with the density differential $d \rho$ thus constructed. Mean convergence of the expansion is to be expected for any square integrable $f(s)$; certain slight restrictions imposed upon $f(s)$ will insure ordinary uniform convergence.

Many authors have written on our subject. My own first approach was based on Hilbert's general theory of spectral decomposition of a bounded symmetric linear operator and specialized it by taking advantage of the particular circumstances prevailing for the dif- 
ferential problem in question, above all, of the fact that for any eigenvalue $\lambda$ the eigenfunction, $\eta(s ; \lambda)$, is known a priori [7]. M. H. Stone's procedure in his book on Linear transformations in Hilbert space [8] is of the same character, but he is able to utilize the machinery of general concepts developed in the twenty intervening years for axiomatized Hilbert space. Earlier, the Stieltjes method, on which Hellinger had founded Hilbert's general theory [9], had been directly applied to the special differential problem by E. Hilb [10]; but he did not carry it so far as to obtain the explicit construction of the differential $d \rho$. Recently E. C. Titchmarsh in several papers and in his book on Eigenfunction expansions [11] resumed this direct approach. The basic equation (12) is due to him. Yet his construction of $w(\lambda)$ and of $d \rho$ is not as direct as I should wish them. Also a number of contributions made by A. Wintner and P. Hartman during the last two years ought to be mentioned [12]. The formula (12) was rediscovered by Kunihiko Kodaira (who of course had been cut off from our Western mathematical literature since the end of 1941); his construction of $\rho$ and his proofs for (12) and the expansion formula (9), still unpublished, seem to clinch the issue. It is remarkable that forty years had to pass before such a thoroughly satisfactory direct treatment emerged; the fact is a reflection on the degree to which mathematicians during this period got absorbed in abstract generalizations and lost sight of their task of finishing up some of the more concrete problems of undeniable importance.

4. Inequalities and asymptotic laws for eigenvalues. But let us drop this matter now and turn to another subject, that of the asymptotic distribution of the eigen-frequencies for the two- or moredimensional membrane and for other oscillating continua. H. A. Lorentz had impressed upon the mathematicians the urgency for physics of a settlement of this question. For a pupil of Hilbert around 1910 it was natural to visualize the question as one concerning integral equations. By means of a real symmetric kernel $K(s, t)$ one introduces the linear operator $u \rightarrow K u$, more explicitly $u(s)$ $\rightarrow \int_{0}^{1} K(s, t) u(t) d t$, in the vector space of all real-valued continuous functions $u=u(s)$ defined over the interval $0 \leqq s \leqq 1$. If the integral $\int_{0}^{1} u(s) v(s) d s$ is taken as the scalar product $(u, v)$ of any two vectors $u, v$ in this space, then the quadratic integral form

$$
K\langle u\rangle=\int_{0}^{1} \int_{0}^{1} K(s, t) u(s) u(t) \cdot d s d t
$$

is the scalar product of $u$ and $K u$. The reciprocal eigenvalues $\kappa$ and 
corresponding eigenvectors $\phi$ are the solutions of the equation $K \phi$ $=\kappa \cdot \phi$. Let the positive $\kappa^{\prime}$ 's be arranged in descending order, $\kappa_{1} \geqq \kappa_{2} \geqq$ $\cdots$, and the corresponding eigenfunctions $\phi_{n}$ so chosen as to form an orthonormal system, $\left(\phi_{m}, \phi_{n}\right)=\delta_{m n}$. Then $\kappa_{n}$ is the maximum of the form $K$ under the auxiliary conditions

$$
\|u\|^{2}=(u, u) \leqq 1, \quad\left(u, \phi_{i}\right)=0 \quad(i=1, \cdots, n-1) .
$$

Let $\omega_{1}(s), \cdots, \omega_{n-1}(s)$ be any $n-1$ functions. The fundamental lemma which made my investigation possible states that there exists a vector $u$ of length $\|u\|=1$ which is orthogonal to $\omega_{1}, \cdots, \omega_{n-1}$,

$$
\left(u, \omega_{1}\right)=0, \cdots,\left(u, \omega_{n-1}\right)=0,
$$

such that $K\langle u\rangle \geqq \kappa_{n}$. This characterizes $\kappa_{n}$ independently of the preceding eigenvalues and eigenfunctions as the "minimum of a maximum." The construction of such a $u$ as the lemma requires is easy enough: a suitable linear combination $c_{1} \phi_{1}(s)+\cdots+c_{n} \phi_{n}(s)$ of the first $n$ eigenvectors will do the trick. I used this lemma (in a slightly different form) for the purpose of carrying over to all $\kappa_{n}$ statements that are evident for the first reciprocal eigenvalue $\kappa_{1}[13]$.

Here is an example. Suppose you add to a kernel $K$ a positive-definite one $k$, that is, one for which $k\langle u\rangle \geqq 0$. It is clear that the first reciprocal positive eigenvalue $\kappa_{1}^{*}$ of $K^{*}=K+k$ is greater than or equal to $\kappa_{1}$; for $\kappa_{1}$ is the maximum of $K\langle u\rangle$ and $\kappa_{1}^{*}$ the maximum of $K\langle u\rangle+k\langle u\rangle$ under the condition $\|u\|^{2} \leqq 1$. Our lemma carries the inequality over to all $\kappa^{\prime}$ s arranged in descending order: $\kappa_{n} \leqq \kappa_{n}{ }^{*}$.

This result is of immediate application to the two-dimensional membrane problem. Let the membrane cover a region $S$ of (Jordan) area $V$. With the argument $P$ ranging over the points of $S$ the eigenvalues $\lambda$ and eigenfunctions $\phi(P)$ satisfy the differential equation $\Delta \phi+\lambda \phi=0$ in $S$ and the condition $\phi=0$ along the boundary $S^{\prime}$ of $S$. The differential equation together with the boundary condition is equivalent to the integral equation

$$
\phi(P)-\lambda \int_{S} G(P, Q) \cdot \phi(Q) \cdot d Q=0,
$$

the symmetric kernel of which is the Green's function $G(P, Q)$. Divide $S$ by a line $l$ into two parts $S_{1}, S_{2}$ and let $G_{1}, G_{2}$ be their Green's functions (setting $G_{i}(P, Q)=0$ if one of the argument points $P, Q$ or both are outside $S_{i}$ ). It can easily be shown that the kernel $G-\left(G_{1}+G_{2}\right)$ is positive-definite. Hence the $n$th eigenvalue $\lambda_{n}$ of $G$ is less than or equal to the $n$th eigenvalue $\lambda_{n}{ }^{\prime}$ of $G_{1}+G_{2}$. The eigenvalues of $G_{1}+G_{2}$ are the eigenvalues of a membrane covering $S$ which 
is kept fixed not only along the boundary $S^{\prime}$, but also along the line $l$. By combining this result with the known asymptotic distribution of the membrane eigenvalues for a square it could readily be deduced that the fixing of the membrane along $l$ does not alter the asymptotic distribution,

$$
\lambda_{n} / \lambda_{n}^{\prime} \rightarrow 1 \quad \text { for } n \rightarrow \infty,
$$

and moreover to establish the law of asymptotic distribution, according to which the number $N(\lambda)$ of eigenvalues less than $\lambda$ equals asymptotically $(V / 4 \pi) \cdot \lambda$.

After the first world war Courant resumed this sort of problems [14]. If I see correctly, his essential contribution is not the minimummaximum principle formulated in our fundamental lemma, but its application to a fairly general typical situation. The maximum of a quadratic form $K\langle u\rangle$ depending on a vector $u$ of length not greater than 1 in a vector space $\mathfrak{S}$ is lowered if additional restrictions are imposed upon $u$, for example, if $u$ is restricted to a linear subspace $\mathfrak{S}^{\prime}$ of $\mathfrak{S}$. If the quadratic form is what Hilbert calls completely continuous, this obvious statement may be put into the inequality $\kappa_{1}^{\prime} \leqq \kappa_{1}$. The quadratic form $K\langle u\rangle$ in $\mathfrak{S}^{\prime}$ is $\left(u, K^{\prime} u\right)$ where $K^{\prime}$ is the operator $K$ followed by perpendicular projection upon $\mathfrak{S}^{\prime}$. While $\kappa_{1}$ is the first reciprocal eigenvalue of the operator $K$ in $\mathfrak{S}, \kappa_{1}^{\prime}$ has the same significance for the operator $K^{\prime}$ in $\mathfrak{S}^{\prime}$. The lemma carries the inequality $\kappa_{1}^{\prime} \leqq \kappa_{1}$ over to all reciprocal (positive) eigenvalues, $\kappa_{n}^{\prime} \leqq \kappa_{n}$. It is clear that the fixing of a membrane along the line $l$ introduces a new restriction, and hence Courant's observation at once gives rise to the inequality $\lambda_{n} \leqq \lambda_{n}^{\prime}$, derived before in another way, for the eigenvalues $\lambda_{n}, \lambda_{n}^{\prime}$ of the undivided and divided membrane. Anyone familiar with the abstract concept of Hilbert space who ponders a little more closely upon the situation to which the principle is applied here, will describe it as follows. All continuous functions $u(P)$ in the closed region $S$ with continuous first derivatives in the interior, such that $u=0$ at the boundary and the Dirichlet integral $D\langle u\rangle=\int_{s}(\operatorname{grad} u)^{2} d P$ is finite, form a functional space $\mathfrak{S}$. Define the square $\|u\|^{2}$ of the length of a vector $u$ in this space by $D\langle u\rangle$, and not by $I\langle u\rangle=\int_{S} u^{2} d P$. The closure of $\mathfrak{E}$ with respect to this metric is a Hilbert space $\overline{\mathfrak{S}}$ and $I\langle u\rangle$ is a completely continuous quadratic form in $\overline{\mathfrak{S}}$. Without altering the other conditions for the functions $u \in \mathfrak{S}$ add the restriction $u=0$ along the line $l$. The closure of the subspace $\mathfrak{S}^{\prime}$ thus obtained is a closed subspace $\overline{\mathfrak{S}^{\prime}}$ of $\overline{\mathfrak{V}}$, and the relation $\kappa_{n}^{\prime} \leqq \kappa_{n}$ holds for the reciprocal eigenvalues of the form $I\langle u\rangle$ in $\overline{\mathfrak{S}}$ and in $\overline{\mathfrak{S}}^{\prime}$ respectively. (Forming the closure is essential since the eigen- 
functions of the divided membrane are in $\overline{\mathfrak{S}}^{\prime}$ but in general not in $\mathfrak{S}^{\prime}$, because of the jump of their normal derivative along $l$. To be sure, by going back to the proof of the fundamental lemma, one can avoid this whole abstract set-up. But for the moment we are interested in the general formulation.)

Let then $K\langle u\rangle$ again be a completeiy continuous quadratic form in a Hilbert space (\$), and $\mathfrak{S}$ be a closed subspace of $(\mathfrak{S}$. For simplicity's sake we assume $K\langle u\rangle$ to be positive-definite and denote the eigenvalues of $K\langle u\rangle$ in $\mathbb{S}$ and in $\mathfrak{S}$ by $\lambda_{n}$ and $\mu_{n}$ respectively. We have seen that

$$
\lambda_{n} \leqq \mu_{n}
$$

Splitting (\$) into $\mathfrak{S}$ and its perpendicular subspace and choosing a basis $p_{1}, p_{2}, \cdots$ for the latter (preferably an orthonormal basis), we can pass from $\mathfrak{S}$ to $\mathfrak{S}$ by a sequence of intermediary subspaces $\mathfrak{S} \subset \mathfrak{S}^{\prime}$ $\subset \mathfrak{S}^{\prime \prime} \subset \cdots, \mathfrak{S}^{(\nu)} \rightarrow$ (S) with $\nu \rightarrow \infty$, by adding one vector of this basis after the other, $\mathfrak{S}^{(v)}=\mathfrak{S}+\left\{p_{1}, \cdots, p_{\nu}\right\}$. Or we can submit the vectors $u$ of $(S)$ to one after the other of the conditions $\left(u, p_{1}\right)=0,\left(u, p_{2}\right)$ $=0, \cdots$, and thus obtain a descending sequence $\left(H \supset S^{\prime} \supset S^{\prime \prime} \supset \cdots\right.$ with $\mathfrak{S f}^{(\nu)} \rightarrow \mathfrak{E}$ for $\nu \rightarrow \infty$. If $\lambda_{n}$ is known, the inequality (13) gives a lower bound for $\mu_{n}$, and the second of our sequences gives rise to an increasing sequence of such lower bounds,

$$
\lambda_{n} \leqq \lambda_{n}^{\prime} \leqq \lambda_{n}^{\prime \prime} \leqq \cdots, \quad \lim _{\nu \rightarrow \infty} \lambda_{n}^{(\nu)}=\mu_{n}
$$

If $\mu_{n}$ is known, the same inequality gives an upper bound for $\lambda_{n}$, which by the first sequence of subspaces may be extended into a whole sequence of decreasing upper bounds, $\mu_{n} \geqq \mu_{n}^{\prime} \geqq \mu_{n}^{\prime \prime} \geqq \cdots \cdot$, $\lim _{\nu \rightarrow \infty} \mu_{n}^{(\nu)}=\lambda_{n}$. With some right we may call the first procedure the Rayleigh-Ritz method and with more right ascribe the second to A. Weinstein [15].

The basic situation encountered here is that of a Hilbert space $\mathfrak{W}$ that splits into a subspace $\mathfrak{S}^{\prime}$ and a perpendicular $\nu$-dimensional space $\left\{p_{1}, \cdots, p_{\nu}\right\}$ spanned by the vector basis $p_{1}, \cdots, p_{\nu}$. N. Aronszajn recently developed two neat formulas concerning this situation [16]. I shall here mention but the one that corresponds to the Weinstein process, the descent from $\mathfrak{S}$ to $\mathfrak{S}^{\prime}$, and omit the other which refers to the inverse Rayleigh-Ritz process. Let $R(\zeta)$ be the resolvent of the operator $K$ in $\mathfrak{S}$, so that $u=R(\zeta) v$ is the solution of the equation $u-\zeta \cdot K u=v$. Moreover let $\lambda^{\prime}, u^{\prime}$ be an eigenvalue and corresponding eigenvector of $K^{\prime}$ in $\mathfrak{S}^{\prime}$. This fact is expressed by the relations 


$$
u^{\prime} \in \mathfrak{S}^{\prime}, \quad u^{\prime}-\lambda^{\prime} \cdot K u^{\prime}=\beta_{1} p_{1}+\cdots+\beta_{\nu} p_{\nu} .
$$

If we suppose that $\lambda^{\prime}$ coincides with no eigenvalue $\lambda_{n}$ of $K$ then the constants $\beta_{i}$ cannot all be zero. By means of the resolvent $R(\zeta)$ the second relation takes on the form $u^{\prime}=\sum_{j=1}^{\nu} \beta_{j} \cdot R\left(\lambda^{\prime}\right) p_{j}$. The first relation requires that the vector $u^{\prime}$ is perpendicular to all the $p_{i}$,

$$
\sum_{j} \beta_{j}\left(p_{i}, R\left(\lambda^{\prime}\right) p_{j}\right)=0 \quad(i, j=1, \cdots, \nu) .
$$

These $\nu$ equations for the $\nu$ unknowns $\beta_{j}$ have a nontrivial solution only if the determinant

$$
\operatorname{det}\left(p_{i}, R(\zeta) p_{j}\right)=W_{p}(\zeta)
$$

vanishes for $\zeta=\lambda^{\prime}$. Divide $W_{p}(\zeta)$ by Gram's determinant $G_{p}$ $=\operatorname{det}\left(p_{i}, p_{j}\right)$. The quotient

$$
W(\zeta)=W_{p}(\zeta) / G_{p}
$$

is clearly independent of the choice of the basis $p_{1}, \cdots, p_{p}$. From the theory of the resolvent one knows that $W(\zeta)$ is a meromorphic function with simple poles at the eigenvalues $\lambda_{n}$ of $K$. On the other hand, we have seen that $W(\zeta)$ vanishes for a value $\zeta=\lambda^{\prime}$ different from all the $\lambda_{n}$ if and only if $\lambda^{\prime}$ is an eigenvalue of $K^{\prime}$ in $\mathfrak{S}^{\prime}$. Hence we shall not find the following equation of Aronszajn too surprising:

$$
W(\zeta)=\left(\frac{-1}{\zeta}\right)^{\nu} \cdot \prod_{n} \frac{\zeta-\lambda_{n}{ }^{\prime}}{\zeta-\lambda_{n}}
$$

I regret that shortness of time prevents me from illustrating these general developments by their applications to the classical problems of elastic and electromagnetic oscillations (D).

5. Zeta-function of the membrane and asymptotic laws for its eigenfunctions. The physicist will not be satisfied with a knowledge of the asymptotic behavior of the eigenvalues alone; also that of the eigenfunctions should be investigated. Carleman was the first to attack this more difficult problem by a new powerful method [17]. Last year we had the good fortune to have with us at the Institute Dr. Åke Pleijel, who had extended Carleman's investigations [18], and Dr. Minakshisundaram, who independently of Carleman had just found a modification of Carleman's method shedding new light on the whole problem. Let us again envisage the two-dimensional membrane. Carleman made use of the Green's function of the "meson" equation $\Delta u-k^{2} u=0$ with the positive parameter $k^{2}$ (for 
the boundary condition $u=0$ ). Minakshisundaram [19] used instead Green's function $G(P, Q ; t)$ of the heat equation

$$
\Delta u-\partial u / \partial t=0
$$

with the positive time parameter $t$.

$$
u(P ; t)=\int_{S} G(P, Q ; t) \cdot f(Q) \cdot d Q
$$

is the temperature of the disk $S$ at the point $P$ and at the moment $t$ if $f(P)$ describes the initial distribution of temperature and the boundary of $S$ is kept constantly on the temperature zero. For the infinite plane $G(P, Q ; t)$ is

$$
G_{0}(P, Q ; t)=\frac{1}{4 \pi t} \cdot \exp \left(-\frac{r_{P Q}^{2}}{4 t}\right)
$$

where $r_{P Q}$ denotes the distance of the two points $P, Q$. For an arbitrary domain $S$ we write

$$
G(P, Q ; t)=G_{0}(P, Q ; t)-g(P, Q ; t) .
$$

For a fixed $P$ in the interior of $S$, the compensating term $g(P, Q ; t)$ is a solution of (14) which vanishes for $t \rightarrow 0$ and on the surface $S^{\prime}$ of $S$ has the same boundary values as the principal part $G_{0}$.

The maximum which the principal term $G_{0}$ assumes when $P$ and $t$ are given, but $Q$ varies over the boundary $S^{\prime}$, is

$$
H_{0}(P ; t)=\frac{1}{4 \pi t} \exp \left(-\frac{l_{P}^{2}}{4 t}\right)
$$

$l_{P}$ denoting the shortest distance of $P$ from the boundary. As a function of $t$ this $H_{0}$ is on the increase from $t=0$ to $t=T=4 / l_{P}^{2}$ and then decreases. A simple argument shows that, as long as $0<t \leqq T$, the compensating function $g(P, Q ; t)$ of $Q$ is positive and reaches its maximum at the boundary, or

$$
0<g(P, Q ; t) \leqq H_{0}(P ; t)
$$

for all $Q$ in $S$ and $0<t \leqq T$.

In terms of the orthonormal system of eigenfunctions $\phi_{n}(P)$ and their eigenvalues $\lambda_{n}$ Green's function is expressible as the sum

$$
\sum_{n} \mathrm{e}^{-\lambda_{n} t} \phi_{n}(P) \phi_{n}(Q)
$$


The series

$$
\zeta(P, Q ; s)=\sum_{n} \frac{\phi_{n}(P) \phi_{n}(Q)}{\lambda_{n}^{s}}
$$

may be called the $\zeta$-function and accordingly (16) the $\theta$-function of the membrane problem. We know that (17) converges uniformly in $P$ and $Q$ for all complex $s$ the real part $\sigma$ of which is greater than or equal to 2 . For $s=1,2,3, \cdots$ the $\zeta$-function gives Green's function $G(P, Q)$ of the membrane and its successive iterations.

One of Riemann's methods for deriving the properties of the ordinary $\xi$-function was based on a connection between the $\theta$-function and the $\zeta$-function, which at once carries over to the general functions here considered as follows:

$$
\Gamma(s) \cdot \zeta(P, Q ; s)=\int_{0}^{\infty} G(P, Q ; t) \cdot t^{8-1} \cdot d t .
$$

On the basis of this relation Minakshisundaram proves that $\Gamma(s)$ $\zeta(P, Q ; s)$ is a regular function of $s$ in the entire $s$-plane if $P, Q$ are two distinct inner points of $S$, but that it is regular except for a simple pole at $s=1$ with residue $1 / 4 \pi$ if $Q$ and $P$ coincide. Still following Riemann, Minakshisundaram splits the integral $\int_{0}^{\infty}$ on the right of (18) into $\int_{0}^{T}+\int_{T}^{\infty}$. Because of the uniform convergence of $\zeta(P, Q ; 2)$, the $\theta$-function (16) falls off exponentially with $t \rightarrow \infty$, and hence the integral

$$
\int_{T}^{\infty} G(P, Q ; t) \cdot t^{8-1} d t
$$

is a regular-analytic function of $s$ in the whole $s$-plane. If $P \neq Q$ the formula

$$
G(P, Q ; t)=\frac{1}{4 \pi t} \exp \left(-\frac{r_{P Q}^{2}}{4 t}\right)-g(P, Q ; t)
$$

together with the estimate (15) proves that $G(P, Q ; t)$ also goes down exponentially to zero with $1 / t \rightarrow \infty$, and hence the integral $\int_{0}^{T}$ is likewise regular-analytic in $s$. This proves the result for $P \neq Q$. However if $Q=P$ then

$$
G(P, P ; t)=\frac{1}{4 \pi t}-g(P, P ; t) .
$$

The second part is positive and does not exceed $H_{0}(P ; t)$; therefore 
$\int_{0}^{T} g(P, P ; t) \cdot t^{o-1} d t$ is regular in $s$, however

$$
\int_{0}^{T} \frac{1}{4 \pi t} t^{s-1} d t=\frac{1}{4 \pi} \cdot \frac{T^{s-1}}{s-1}
$$

has a pole at $s=1$ with the residue $1 / 4 \pi$. That completes the proof. $\zeta(P, Q ; s)$ itself has zeros for the same values for which the regular function $1 / \Gamma(s)$ has zeros, namely for $s=0,-1,-2, \cdots$.

Integration over $P$, one might think, would give for

$$
\zeta(s)=\int_{S} \zeta(P, P ; s) \cdot d P=\sum_{n} \lambda_{n}^{-\bullet}
$$

the following result

$$
\Gamma(s) \cdot \zeta(s)=\frac{V}{4 \pi} \cdot \frac{1}{s-1}+\text { a regular function } R(s)
$$

Unfortunately this conclusion is too hasty: if $P$ is near the boundary, $H_{0}(P ; t)$ is not so small. By carrying out the integration over $P$ in the relation

$$
G(P, P ; t)=\frac{1}{4 \pi t}-g(P, P ; t), \quad 0 \leqq g(P, P ; t) \leqq H_{0}(P ; t),
$$

one finds that the remainder $R(s)$ in (19) is regular at least for $\sigma>1 / 2$; it seems difficult to go beyond the vertical $\sigma=1 / 2$.

Standard devices familiar from the theory of Riemann's $\zeta$-function permit one to deduce from this behavior of the $\zeta$-function of the membrane Carleman's asymptotic formulas

$$
\sum_{\lambda_{n} \leqq \lambda}\left(\phi_{n}(P)\right)^{2} \sim \lambda / 4 \pi, \quad N(\lambda)=\sum_{\lambda_{n} \leqq \lambda} 1 \sim V \lambda / 4 \pi
$$

and also the "incoherence relation"

$$
\sum_{\lambda_{n} \leqq \lambda} \phi_{n}(P) \phi_{n}(Q)=o(\lambda)
$$

I feel that these informations about the proper oscillations of a membrane, valuable as they are, are still very incomplete. I have certain conjectures on what a complete analysis of their asymptotic behavior should aim at; but since for more than 35 years I have made no serious attempt to prove them, I think I had better keep them to myself.

In general, it can not be expected that our $\zeta$-function satisfies a functional equation of the Riemann type; one may guess that this 
feature depends on the homogeneity of the domain of integration. Such a domain is the circumference of the unit circle. Functions on it are functions $f(x)$ of period $2 \pi$. The periodic eigenfunctions $\phi$ and corresponding eigenvalues $\lambda$ of $d^{2} \phi / d x^{2}+\lambda \phi=0$ are

$$
\phi(x)=\mathrm{e}^{i n x}, \quad \lambda_{n}=n^{2} \quad(n=0, \pm 1, \pm 2, \cdots) .
$$

This leads straight to the Riemann $\zeta$-function $\sum_{n=1}^{\infty} n^{-2 s}$ usually denoted not by $\zeta(s)$ but by $\zeta(2 s)$. It was therefore natural that Minakshisundaram should investigate the spherical harmonics on a $k$-dimensional sphere (in $k+1$-dimensional space). Here he found indeed a sort of Riemann functional equation, the structure of which is, however, essentially more complicated than in the classical Riemann case $k=1$.

The two-sphere is homogeneous because it permits a compact transitive Lie-group $\sigma$ of transformations $s$ into itself, namely the group of rotations. The spherical harmonics of order $l$ form a $(2 l+1)$ dimensional linear manifold that is invariant with respect to the group of rotations and has the property of irreducibility in this regard. Consider arbitrary (complex-valued) continuous functions on the sphere and define the scalar product of two such functions $f$ and $g$ by the integral $\int \bar{g}(P) f(P) \cdot d \omega_{P}$ formed by means of the invariant area element $d \omega_{P}$. It is obvious how to generalize this situation to any homogeneous manifold $S$ of points $P$, that is, any manifold that permits a compact transitive Lie-group $\sigma$ of transformations $s, P \rightarrow s P$. The existence of an invariant volume element on such a manifold (which itself is of necessity compact) follows easily from the fact that a compact Lie-group has an invariant volume element $d s$. We normalize the unit for measuring volumes on the group so that the total volume $\int d s$ of the group becomes 1 . The integrals with respect to $s$ are then in truth mean values. The transform $s f$ of a function $f=f(P)$ on $S$ is defined by $s f(s P)=f(P)$ or $s f(P)=f\left(s^{-1} P\right)$. A set $\phi_{1}(P), \cdots$, $\phi_{h}(P)$ of functions on $S$, or the manifold of their linear combinations $\phi(P)=x_{1} \cdot \phi_{1}(P)+\cdots+x_{h} \cdot \phi_{h}(P)$, is invariant if each $s \phi_{i}(P)$ is a linear combination $\sum_{j} \omega_{j i}(s) \cdot \phi_{i}(P)$ of the $\phi_{i}$ themselves. Then $s \rightarrow\left\|\omega_{i j}(s)\right\|$ is a representation of degree $h$ of the group $\sigma$. Inequivalent irreducible invariant sets are orthogonal to each other. Besides orthogonality there is the completeness relation, to which we shall presently return. Thus we are in possession of the "eigenfunctions" of the homogeneous manifold, the sequence of which is subdivided into irreducible invariant sets of finite length. Theorems about summability of expansions in terms of these eigenfunctions have been proved by S. Bochner [20]. But so far they are eigenfunctions with- 
out eigenvalues. It was the young Dutch physicist H. B. G. Casimir who, prompted by the applications of group theory to quantum mechanics, found the eigenvalues. Indeed he constructed an invariant self-adjoint differential operator $\Delta$ working on arbitrary functions $f(P)$ which is the analogue of the Laplace operator on a sphere, and he was able to show that the functions of a given irreducible invariant set satisfy an equation $\Delta \phi+\lambda \phi=0$ with a constant $\lambda$ characteristic for the entire set [21]. Having the eigenfunctions, one can, following a suggestion by Bochner, form the $\zeta$-function

$$
\zeta(P, Q ; z)=\sum_{n} \frac{\phi_{n}(P) \phi_{n}(Q)}{\lambda_{n}^{s}}
$$

and might expect that this function, in addition to having the properties quite generally established by Minakshisundaram, will satisfy a functional equation of Riemann's type. But this is a question that remains to be investigated.

6. Integral equations and the group-theoretic completeness relation. The proof of the completeness relation for invariant sets on a homogeneous manifold $S$ is one of the most surprising applications of the eigenvalue theory of integral equations. If the manifold $S$ is the compact Lie group itself under the influence of its left translations, then this theorem states the completeness of the totality of all irreducible representations of the group. But the method for its proof, developed in 1927 by F. Peter and the speaker [22], not only carries over to the homogeneous manifolds, but applies to a far more general situation, that is best described in axiomatic terms [23]. We replace the functions on the homogeneous manifold by vectors $f$ in a vector space $\Sigma$ and suppose that $\Sigma$ bears a Hermitian metric defined by a scalar product $(g, f)$ with the usual properties including the positive character of $(f, f)=\|f\|^{2}$. An abstract compact Lie group $\sigma$ is given and a representation of its elements $s$ by linear transformations $f \rightarrow s f$ in our vector space. The invariance of the metric is assumed, $(s g, s f)$ $=(g, f)$. Let $f$ be a given vector. All vectors that will occur in our construction are prepared from $f$ by forming linear combinations of its transforms $s f$. Besides $\Sigma$ we envisage the "vector space" $\Xi$ of all continuous functions $\xi=\xi(s)$ on the group manifold $\sigma$ and define a linear mapping $\xi \rightarrow g$ of $\boldsymbol{\Xi}$ onto $\Sigma$ by

$$
g=\mathfrak{f} \xi=\int \xi(s) \cdot s f \cdot d s,
$$

and its Hermitian conjugate, a mapping $g \rightarrow \xi$ of $\Sigma$ onto $\Xi$, by 


$$
\xi=f^{*} g=(s f, g) .
$$

The mapping $\mathfrak{f}^{*} \mathfrak{f}=\mathfrak{F}$ of $\Xi$ into itself has the positive-definite Hermitian kernel $H(s, t)=(s f, t f)$. By Erhard Schmidt's method we construct its largest reciprocal eigenvalue $\gamma$ and an orthonormal set of eigenfunctions $\phi_{1}(s), \cdots, \phi_{h}(s)$ for it. Repetition of the construction gives the reciprocal eigenvalues in descending order, $\gamma>\gamma^{\prime}>\cdots$, and the sought-for completeness relation results from the well known fact that the trace of $H$ equals the sum of the reciprocal eigenvalues,

$$
\|f\|^{2}=(f, f)=h \gamma+h^{\prime} \gamma^{\prime}+\cdots .
$$

Indeed $\int H(s, t) \cdot \phi_{i}(t) \cdot d t=\gamma \cdot \phi_{i}$ may be written in the form

$$
\mathfrak{f} \phi_{i}=\gamma^{1 / 2} \cdot g_{i}, \quad f^{*} g_{i}=\gamma^{1 / 2} \cdot \phi_{i}
$$

where the first equation is to be taken as the definition of the vector $g_{i}$. The $g_{i}$ then form an orthonormal invariant set, and if the Fourier coefficients $\left(g_{i}, f\right)=\alpha_{i}$ are introduced one finds that

$$
h \gamma=\left|\alpha_{1}\right|^{2}+\cdots+\left|\alpha_{h}\right|^{2} .
$$

Thus the completeness relation follows, stating that the orthonormal sequence $g_{1}, g_{2}, \cdots,\left(g_{m}, g_{n}\right)=\delta_{m n}$, resulting from our construction and consisting of sections of finite length, each of which is an invariant set, makes the absolute square sum $\left|a_{1}\right|^{2}+\left|\alpha_{2}\right|^{2}+\cdots$ of the Fourier coefficients $\alpha_{i}=\left(g_{i}, f\right)$ not only $\leqq\|f\|^{2}$, as is trivial (Bessel's inequality), but actually $=\|f\|^{2}$. The construction picks out those invariant sets that contribute to $f$ and $\|f\|^{2}$.

This feature is quite essential when, with Harald Bohr and J. von Neumann [24], all restrictions concerning the group $\sigma$ are abandoned. The construction still works, provided one supposes $f$ to be "almost periodic." But in general there are under these circumstances more than denumerably many inequivalent irreducible invariant sets of vectors; but $f$ itself picks out those among them that matter for $f$.The assumption of almost periodicity is highly restrictive. One may instead impose some slight restriction on the group, e.g., local compactness, and at the same time admit a far wider class of vectors $f$. In that case nothing resembling completeness is to be expected unless one includes also representations of infinite degree. This step has recently been taken by D. Rykov and I. Gelfand in Russia, by V. Bargmann and I. Segal in this country [25].

I think it is time for me to stop here. I have not even touched on the extension of Hilbert's theory of bounded to non-bounded linear operators, which came about under the pressure of quantum me- 
chanics, nor to the connection between spectral decomposition and ergodic theory. Other mathematicians at other times have spoken or will speak on these subjects with more competence than I could. I hope you have taken this lecture for what it was meant to be: a Plauderei, the chat of a man who has reached the age where it is more pleasant to remember the past than to look forward into the future. Even so, it gives him a little satisfaction to see that the issues to which the efforts of his youth were dedicated have kept alive over the years and are still in the process of unfolding their implications.

\section{Notes}

(A) There are two classes of eigenfunctions. But since one of them does not contribute to the expansion of the discontinuous function $1^{0}(x)$, only the eigenvalues $\lambda^{2}$ belonging to the other class have to be taken into account; they are determined by the transcendental equation

$$
\beta \cdot \tan \frac{\alpha \lambda \pi}{2}+\alpha \cdot \tan \frac{\beta \lambda \pi}{2}=0 .
$$

It is easily seen that for every integer $n$ this equation has exactly one root $\lambda_{n}$ of the form $\lambda_{n}=2 n+\theta_{n},-1<\theta_{n}<1$. If $\alpha$ and $\beta$ are rational,

$$
\alpha=a / c, \quad \beta=b / c, \quad a+b=c ; a, b, c \text { integers, } c>0,
$$

then $\theta_{n}$ has the period $c, \theta_{n+c}=\theta_{n}$, and this circumstance makes a fairly explicit evaluation of the $n$th partial sum $1_{n}^{0}(x)$ possible.

(B) The main fact is as follows: The given values $\beta_{1}=w\left(\alpha_{1}\right)$, $\beta_{2}=w\left(\alpha_{2}\right), \cdots$ have to satisfy a sequence of inequalities of which the first, $\Im \beta_{1}>0$, involves only $\beta_{1}$, the second $\beta_{1}$ and $\beta_{2}$, and so on. If these inequalities are fulfilled then there are two possibilities, which are distinguishable by a convergence criterion. In the first, the limit point case, the problem has a unique solution; in the second, the limit circle case, the manifold of all solutions $w(\lambda)$ is obtained from that of all positive functions $z(\lambda)$ by a certain Möbius transformation

$$
w(\lambda)=\frac{A(\lambda) \cdot z(\lambda)+B(\lambda)}{C(\lambda) \cdot z(\lambda)+D(\lambda)}
$$

with coefficients $A, B, C, D$ that are regular analytic functions of $\lambda$ in the upper half $\lambda$-plane.

(C) Indeed replacing $\lambda_{0}, \lambda$ by $\lambda, \bar{\lambda}$ in (6) one finds

$$
\Im \phi(s)=\Im \lambda \cdot \int_{0}^{l} G(s, t) \cdot \Phi(t) \cdot d t
$$


and therefore $\Im \phi(s)$ has a uniformly convergent expansion in terms of the eigenfunctions $\eta\left(s ; \lambda_{n}\right)$. For the integral $\int_{0}^{i} \phi(s) \eta\left(s ; \lambda_{n}\right) \cdot d s$ one obtains from (3) the value $1 /\left(\lambda_{n}-\lambda\right)$.

(D) The eigenvalues $\lambda=\mu_{n}$ of $\Delta u+\lambda u=0$ corresponding to the boundary condition $\partial u / \partial n=0$ (normal derivative of $u$ equal to zero; "acoustic eigenvalues") are the reciprocals of the successive maxima of $I\langle u\rangle$ under the restriction $D\langle u\rangle=1$, or the successive minima of $D\langle u\rangle$ under the restriction $I\langle u\rangle=1$. The boundary condition $\partial u / \partial n=0$ gets lost, as it were, in the process of closure under the metric defined by $D\langle u\rangle$. Hence $\mu_{n} \leqq \lambda_{n}$, where $\lambda_{n}$, as before, are the membrane eigenvalues corresponding to the boundary condition $u=0$.

The equation for the oscillations of a plate,

$$
\Delta \Delta u-\lambda^{2} \cdot u=0
$$

arises from minimizing

$$
\int_{S}(\Delta u)^{2} \cdot d P
$$

under the auxiliary condition $I\langle u\rangle=1$. Let $\mu_{n}^{2}$ be the eigenvalues of the clamped plate,

$$
\text { boundary conditions } u=0, \quad \frac{\partial u}{\partial n}=0,
$$

and $\lambda_{n}^{2}$ those for the "half-free" plate,

$$
\text { boundary conditions } u=0, \quad \Delta u=0 .
$$

Again the boundary condition $\Delta u=0$ gets lost in the process of closure under the metric defined by (20). Hence $\lambda_{n}^{2} \leqq \mu_{n}^{2}$; one can further expect that $\lambda_{n}$ and $\mu_{n}$ follow the same asymptotic law. Weinstein observed that the eigenvalues of the half-free plate (as their notation indicates) are simply the squares of the membrane eigenvalues. Indeed if $\Delta \Delta u=\lambda^{2} u(\lambda>0)$ set $\Delta u=-\lambda v$, so that $\Delta v+\lambda u=0$. The boundary conditions (21) give $u=0, v=0$ along $S^{\prime}$, hence $(u+v) / 2$ is a membrane eigenfunction with the eigenvalue $\lambda$ and $(u-v) / 2$ for $-\lambda$. But the membrane has no negative eigenvalues; consequently $u-v=0$ and $(u+v) / 2=u$.

It was by a somewhat similar remark that I had previously reduced the elastic oscillations of a three-dimensional body asymptotically to the three-dimensional membrane problem [26]. The vector field $\mathfrak{v}(P)$ describing a proper oscillation of the elastic body satisfies an 
equation

$$
a \cdot \operatorname{grad} \operatorname{div} \mathfrak{v}-b \cdot \operatorname{rot} \operatorname{rot} \mathfrak{v}+\lambda \mathfrak{v}=0
$$

in $S$

with two elastic constants $a, b$. Impose the boundary conditions

$\mathfrak{v}$ normal, $\operatorname{div} \mathfrak{v}=0$ on the surface $S^{\prime}$ of $S$.

For $\phi=\operatorname{div} \mathfrak{b}$ one finds $a \cdot \Delta \phi+\lambda \phi=0$ in $S$ and the boundary condition $\phi=0$; hence if $\phi$ is an eigenfunction of the membrane problem with the eigenvalue $\lambda / a$ then $\mathfrak{v}=\operatorname{grad} \phi$ satisfies $(E),\left(E^{\prime}\right)$. If, however, $\operatorname{div} \mathfrak{v}=0$ throughout $S$ we have

$$
b \cdot \Delta \mathfrak{v}+\lambda \mathfrak{b}=0, \quad \operatorname{div} \mathfrak{v}=0 \text { in } S ; \quad \mathfrak{b} \text { normal on } S^{\prime}
$$

(since $\Delta \mathfrak{v}=\operatorname{grad} \operatorname{div} \mathfrak{v}-\operatorname{rot} \operatorname{rot} \mathfrak{b}$ ), or $\lambda / b$ is an eigenvalue of the problem of radiation in a Hohlraum $S$ whose wall $S^{\prime}$ is a perfect mirror. Denoting the numbers of eigenvalues $\leqq \lambda$ of the membrane, the radiation, and the elastic problem (E) \& $\left(\mathrm{E}^{\prime}\right)$ by $N_{m}(\lambda), N_{r}(\lambda)$ and $N_{e}(a, b ; \lambda)$ respectively, we thus find the relation

$$
N_{\bullet}(a, b ; \lambda)=N_{m}(\lambda / a)+N_{r}(\lambda / b) .
$$

For $a=b=1$ the left side of (E) turns into $\Delta \mathfrak{b}+\lambda \mathfrak{b}$. Since asymptotically the boundary conditions are of no influence we must have the asymptotic relation

$$
N_{e}(1,1 ; \lambda) \sim 3 N_{m}(\lambda), \quad \text { that is, } N_{m}(\lambda)+N_{r}(\lambda) \sim 3 N_{m}(\lambda)
$$

or $N_{r}(\lambda) \sim 2 N_{m}(\lambda)$ and thus

$$
N_{e}(a, b ; \lambda) \sim N_{m}(\lambda / a)+2 N_{m}(\lambda / b) .
$$

\section{BIBLIOGRAPHY}

1. H. Weyl, Die Gibbs'sche Erscheinung in der Theorie der Kugelfunktionen, Rend. Circ. Mat. Palermo vol. 29 (1910) pp. 308-328; Ueber die Gibbs'sche Erscheinung und verwandte Konvergenzphänomene, Rend. Circ. Mat. Palermo vol. 30 (1910) pp. $1-31$.

2. - Ueber die Gleichverteilung von Zahlen mod. Eins, Math. Ann. vol. 77 (1916) pp. 313-352.

3. - Ueber gewöhnliche Differentialgleichungen mit Singularitatten und die zugehörigen Entwicklungen willkiurlicher Funktionen, Math. Ann. vol. 68 (1910) pp. 220-269; Ueber gewöhnliche Differentialgleichungen mit singulären Stellen und ihre Eigenfunktionen, Nachr. Ges. Wiss. Göttingen (1910) pp. 442-467.

4. E. Hellinger, Zur Stieltjes'schen Kettenbruchtheorie, Math. Ann. vol. 86 (1922) pp. 18-29.

5. G. Pick, Ueber die Beschränkungen analytischer Funktionen, welche durch vorgegebene Funktionswerte bewirkt werden, Math. Ann. vol. 77 (1916) pp. 7-23. R. Nevanlinna, Ueber beschränkte Funktionen, die in gegebenen Punkten vorgeschriebene Werte 
annehmen, Annales Academiae Scientiarium Fennicae vol. 13, No. 1, 1919; Ueber beschränkte analytische Funktionen, ibid. vol. 32, No. 7, 1929.

6. H. Weyl, Ueber das Pick-Nevanlinna'sche Interpolationsproblem und sein infinitesimales Analogon, Ann. of Math. vol. 36 (1935) pp. 230-254.

7. - Chapter 3 of the first, and $\$ 1$ of the second, paper quoted under [3].

8. M. H. Stone, Linear transformations in Hilbert space, Amer. Math. Soc. Colloquium Publications, vol. 15, 1932.

9. E. Hellinger, Neue Begrïndung der Theorie quadratischer Formen von unendlichvielen Veränderlichen, J. Reine Angew. Math. vol. 136 (1909) pp. 265-326.

10. E. Hilb, Ueber gewöhnliche Differentialgleichungen mit Singularitäten und die dazugehörigen Entwicklungen willkitrlicher Funktionen, Math. Ann. vol. 76 (1915) pp. 333-339.

11. E. C. Titchmarsh, Eigenfunction expansions associated with second-order differential equations, Oxford, Clarendon Press, 1946.

12. A. Wintner, Stability and spectrum in the wave mechanics of lattices, Physical Review vol. 72 (1947) pp. 81-82; On the normalization of characteristic differentials in continuous spectra, ibid. vol. 72 (1947) pp. 516-517; On the location of continuous spectra, Amer. J. Math. vol. 70 (1948) pp. 22-30; Asymptotic integrations of the adiabatic oscillator, Amer. J. Math. vol. 69 (1947) pp. 251-272, and Duke Math. J. vol. 15 (1948) pp. 53-67. P. Hartmann and A. Wintner, An oscillation theorem for continuous spectra, Proc. Nat. Acad. Sci. U.S.A. vol. 33 (1947) pp. 376-379.

13. H. Weyl, Das asymptotische Verteilungsgesetz der Eigenwerte linearer partieller Differentialgleichungen, Math. Ann. vol. 71 (1911) pp. 441-469; Ueber die Abhängigkeit der Eigenschwingungen einer Membran von deren Begrenzung, J. Reine Angew. Math. vol. 141 (1912) pp. 1-11.

14. See his account in: R. Courant and D. Hilbert, Methoden der mathematischen Physik, vol. 1, 2d ed., Berlin, 1931, in particular Chap. 6, \$4.

15. A. Weinstein, Étude des spectres des équations aux dérivées partielles de la théorie des plaques élastiques, Mémorial des Sciences Mathématiques, vol. 88, 1937.

16. N. Aronszajn, Rayleigh-Ritz and A. Weinstein methods for approximation of eigenvalues, I: Operators in a Hilbert space, Proc. Nat. Acad. Sci. U.S.A. vol. 34 (1948) pp. 474-480; II: Differential operators, ibid. pp. 594-601.

17. T. Carleman, Propriétés asymptotiques des fonctions fondamentales des membranes vibrantes, Förhandlingar Skandinaviska Matematikerkongressen Stockholm, 1934, pp. 34-44; Ueber die asymptotische Verteilung der Eigenwerte partieller Differentialgleichungen, Berichte über die Verhandlungen der Sächsischen Akademie der Wissenschaften zu Leipzig vol. 88 (1936) pp. 119-132.

18. Å. Pleijel, Propriêtés asymptotiques des fonctions et valeurs propres de certains problèmes de vibration, Arkiv för Matematik, Astronomi och Fysik vol. 27 A, No. 13, 1940; Sur la distribution des valeurs propres de problèmes régis par l'équation $\Delta u$ $+\lambda k(x, y) u=0$, ibid. vol. 29 B, No. 7, 1943; On Hilbert-Schmidt's theorem in the theory of partial differential equations, Fysiografiska Sälskapets i Lund Förhandlingar vol. 17, No. 2, 1946; Asymptotic relations for the eigenfunctions of certain boundary problems of polar type, Amer. J. Math. vol. 70 (1948) pp. 892-907.

19. S. Minakshisundaram, $A$ generalization of Epstein zeta functions, Canadian Journal of Mathematics vol. 1 (1949) pp. 320-327. S. Minakshisundaram and Å Pleijel, Some properties of the eigenfunctions of the Laplace-operator on Riemannian manifolds, Canadian Journal of Mathematics vol. 1 (1949) pp. 242-256.

20. S. Bochner, Summation of derived Fourier series (An application to Fourier expansions on compact Lie groups), Ann. of Math. vol. 37 (1936) pp. 345-356. 
21. H. B. G. Casimir, Rotation of a rigid body in quantum mechanics, Leiden thesis, 1931.

22. F. Peter and H. Weyl, Die Vollständigkeit der primitiven Darstellungen einer geschlossenen kontinuierlichen Gruppe, Math. Ann. vol. 97 (1927) pp. 737-755.

23. $\mathrm{H}$. Weyl, Almost periodic invariant vector sets in a metric vector space, Amer. J. Math. vol. 71 (1949) pp. 178-205.

24. I quote but the two most important papers: H. Bohr, Zur Theorie der fastperiodischen Funktionen I, Acta Math. vol. 45 (1925) pp. 29-127. J. von Neumann, Almost periodic functions in a group I, Trans. Amer. Math. Soc. vol. 36 (1934) pp. 445492. For further references see [23].

25. I. Gelfand and D. Rykov, Irreducible unitary representations of locally compact groups, Rec. Math. (Mat. Sbornik) N. S. vol. 3 (1943) pp. 301-316; also: Academy of Sciences of the USRR. Journal of Physics vol. 10 (1946) pp. 93-94. V. Bargmann, Irreducible unitary representations of the Lorentz group, Ann. of Math. (2) vol. 48 (1947) pp. 568-640. I. E. Segal, Irreducible representations of operator algebras, Bull. Amer. Math. Soc. vol. 53 (1947) pp. 73-88.

26. $\mathrm{H}$. Weyl, Das asymptotische Verteilungsgesetz der Eigenschwingungen eines beliebig gestalteten elastischen Körpers, Rend. Circ. Mat. Palermo vol. 39 (1915) pp. 1-49.

INSTITUTE fOR Advanced STUdy 